\title{
Kikuchi-Fujimoto disease in the regional lymph nodes with node metastasis in a patient with tongue cancer: A case report and literature review
}

\author{
TESSHO MARUYAMA ${ }^{1,2}$, KAZUHIDE NISHIHARA $^{1,2}$, MASANAO SAIO $^{3,4}$, TOSHIYUKI NAKASONE $^{2}$, \\ FUMIKAZU NIMURA $^{1}$, AKIRA MATAYOSHI ${ }^{2}$, TAKAHIRO GOTO ${ }^{2}$, NAOKI YOSHIMI $^{3}$ and AKIRA ARASAKI ${ }^{1,2}$ \\ ${ }^{1}$ Department of Oral and Maxillofacial Functional Rehabilitation, Graduate School of Medicine, University of the Ryukyus; \\ Departments of ${ }^{2}$ Oral and Maxillofacial Surgery, and ${ }^{3}$ Pathology, Ryukyu University Hospital; ${ }^{4}$ Department of Pathology \\ and Oncology, Graduate School of Medicine, University of the Ryukyus, Nishihara, Okinawa 903-0215, Japan
}

Received July 27, 2016; Accepted March 9, 2017

DOI: 10.3892/ol.2017.6139

\begin{abstract}
Kikuchi-Fujimoto disease (KFD), also known as histiocytic necrotizing lymphadenitis, is a rare self-limiting disorder typically affecting the cervical lymph nodes (LNs), which is often misdiagnosed as other LN-associated diseases. KFD frequently presents with necrotic lesions and recurrences, which are also features of metastatic LNs. Clinicians may thus suspect LN metastasis when they encounter ipsilateral cervical lymphadenopathy in a patient with head and neck cancer. The present study reports the case of a 48-year-old man with tongue cancer and KFD affecting the right edge of his tongue and ipsilateral cervical LNs. LN metastasis was initially suspected, but pathological examination of the dissected LNs revealed one necrotic metastatic lesion and two necrotic KFD lesions. Ipsilateral cervical lymphadenopathy recurred 6 years after the initial surgery, and it was not possible to differentiate clinically between a second primary tumor and recurrent KFD prior to treatment. To the best of our knowledge, this is the first reported case of simultaneous tongue cancer, regional LN metastasis and KFD. This highlights the requirement to consider KFD in the event of LNs with necrotic lesions but no cancerous cells. A combination of clinical and pathological approaches may aid in the diagnosis of KFD, in addition to ruling out $\mathrm{LN}$
\end{abstract}

Correspondence to: Dr Kazuhide Nishihara, Department of Oral and Maxillofacial Functional Rehabilitation, Graduate School of Medicine, University of the Ryukyus, 207 Uehara, Nishihara, Okinawa 903-0215, Japan

E-mail: kazuhide@med.u-ryukyu.ac.jp

Abbreviations: KFD, Kikuchi-Fujimoto disease; LN, lymph node; CT, computed tomography; US, ultrasound; MRI, magnetic resonance imaging; SCC, squamous cell carcinoma; $\mathrm{CD}$, cluster of differentiation; FDG-PET, 2-[18F]-fluoro-2-deoxy-D-glucose positron emission tomography

Key words: Kikuchi-Fujimoto disease, lymph node metastasis, necrosis, recurrence, pathology, clinical, tongue, cancer metastasis in initial and recurrent lymphadenopathies. The present study indicate that a diagnosis of KFD should be considered in patients with head and neck cancer that exhibit necrotic LNs lacking cancerous cells. This is important, as misdiagnosis of KFD as LN metastasis may lead to unnecessary adjuvant therapy.

\section{Introduction}

Kikuchi-Fujimoto disease (KFD), also known as histiocytic necrotizing lymphadenitis, was first described in the Japanese literature in 1972 (1,2). Up until 2013, 733 patients with KFD have been reported (3). Most patients with KFD present with localized unilateral cervical lymphadenopathy (axillary and inguinal are infrequently) with or without pain and fever (4). KFD occurs worldwide (5) with a relatively high prevalence, particularly among young women and girls in Japan, as well as in other Asian countries (6). KFD is a rare but well known self-limiting disorder that typically affects the cervical lymph nodes (LNs), with a possibility of recurrence $(6,7)$. However, the etiology of KFD remains unknown, and its clinical features of lymphadenopathy and necrotic lesions are frequently misdiagnosed as other LN diseases, including malignancy. Metastatic LNs of cancer also generally demonstrate necrosis and recurrence; thus, clinicians may suspect LN metastasis in patients with ipsilateral cervical lymphadenopathy and head and neck cancer. Metastatic LNs may exhibit central necrosis, particularly in cases of advanced metastasis, and preoperative therapy for cancer may also cause LN necrosis. Regional recurrence of neck metastases is common in patients with head and neck cancer.

The present study reports the case of a patient with tongue cancer and ipsilateral cervical lymphadenopathy. Pathological examination revealed two types of lesions in three nodes, including one metastatic lesion and two KFD lesions, all with evidence of necrosis. Ipsilateral cervical lymphadenopathy recurred 6 years after the initial surgery, and clinical examination was unable to differentiate between the second primary tumor and KFD. 


\section{Case report}

A 48-year-old man visited the Department of Oral and Maxillofacial Surgery, University Hospital of the Ryukyus (Okinawa, Japan) in April 2008 for treatment of a tongue mass. The patient had a 2-month-long history of pain associated with the right lateral tongue edge. Physical examination revealed a hard elastic $2.0 \times 1.5 \mathrm{~cm}$ mass on the right side of the tongue (Fig. 1). Initial examination revealed no palpable lymphadenopathy in the head and neck area. The patient's medical history was unremarkable, except for Cushing's syndrome and glaucoma. Initial contrast-enhanced computed tomography (CT) with iopamidol (Oypalomin 370; Fuji Pharma, Tokyo, Japan), ultrasound (US) and magnetic resonance imaging (MRI) identified no cervical LN lesions. Incisional biopsy of the tongue mass (May 2008) led to a histrogical diagnosis of squamous cell carcinoma (SCC) (cT2NOM0) (8). Considering the patient's young age, intravenous neoadjuvant bleomycin was administered ( $15 \mathrm{mg} /$ day for 6 days; total dose $90 \mathrm{mg}$ ) plus oral uracil/tegafur (450 mg/day) as previously described $(9,10)$. The tongue mass partially responded to preoperative chemotherapy, based on revised RECIST guidelines (version 1.1) (11). However, ipsilateral cervical lymphadenopathy developed several days prior to the scheduled surgery. One LN at level I was mildly enlarged, firm, round, movable and painless on palpation, suggesting metastasis. The other LNs exhibited no signs of metastasis. Subsequent CT was performed to reevaluate the clinical stage. The level I LN appeared as a $1.2 \times 0.9 \mathrm{~cm}$ round mass with central necrosis and rim enhancement, suggesting LN metastasis. However, CT also revealed two enlarged oval-shaped LNs of $\geq 1.0 \mathrm{~cm}$ at levels II and III, with homogeneous contrast enhancement (Fig. 2), which could not be conclusively diagnosed as cancerous. A clinical diagnosis of tongue SCC (cT2N1M0) was made using UICC TNM classification 7th edition (8).

Radical neck dissection was performed (May 2008) with dissection and pathological diagnosis of LNs at all levels. The patient simultaneously underwent local excision of the tongue cancer. Histopathological examination revealed tongue SCC with one metastatic node at level I with necrosis (Fig. 3; a $3-\mu \mathrm{m}$ section was cut and stained with hematoxylin and eosin and evaluated with brightfield microscopy), with no extracapsular nodal spread or positive margins. The level II and III LNs revealed necrotizing granulomatous lesions, and the presence of coagulative necrosis surrounded by numerous epithelioid cells accompanying nuclear debris, clinical featured of KFD (Fig. 4; a 3- $\mu$ m section was cut and stained with hematoxylin and eosin and evaluated with brightfield microscopy). Immunohistochemical analysis was performed with the following antibodies: AE1/AE3 (dilution, 1:800; catalog no. M3515; Dako; $100^{\circ} \mathrm{C} / 60$ min of incubation); cluster of differentiation (CD) 3 (dilution, 1:50; catalog no. M7254; Dako; $100^{\circ} \mathrm{C} / 60$ min of incubation); CD20 (dilution, 1:200; catalog no. M0755; Dako; $100^{\circ} \mathrm{C} / 60$ min of incubation); CD68 (dilution, 1:1,600; catalog no. M0814; Dako; room temperature/8 min of incubation). Immunohistochemical examination for the pancytokeratin marker AE1/AE3 revealed no epithelial component, indicating that the lesion was not a tumor metastasis (data not shown). No abnormal distributions of $\mathrm{CD}^{+} / \mathrm{CD} 20^{+}$ lymphocytes were identified (data not shown). Detection of the histiocyte marker CD68 confirmed the epithelioid cells as

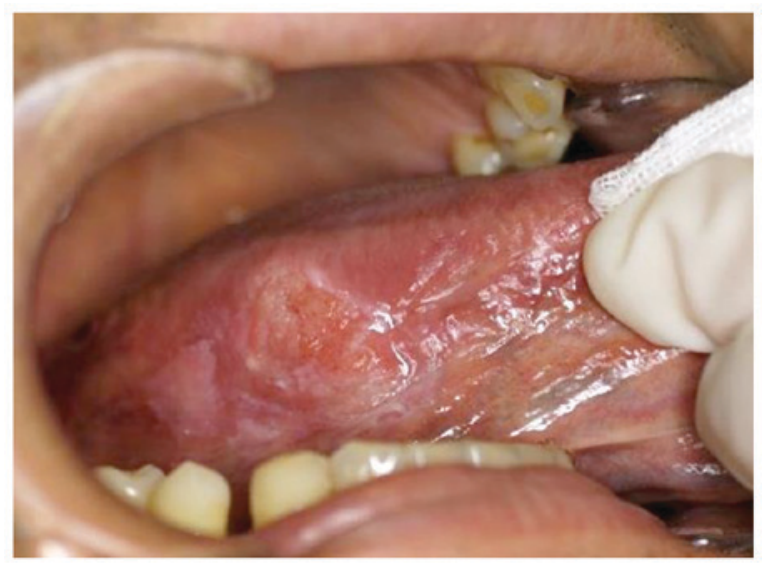

Figure 1.Intraoral image of the lesion captured at the initial visit. Physical examination revealed a hard elastic $2.0 \times 1.5 \mathrm{~cm}$ mass on the right side of the tongue.

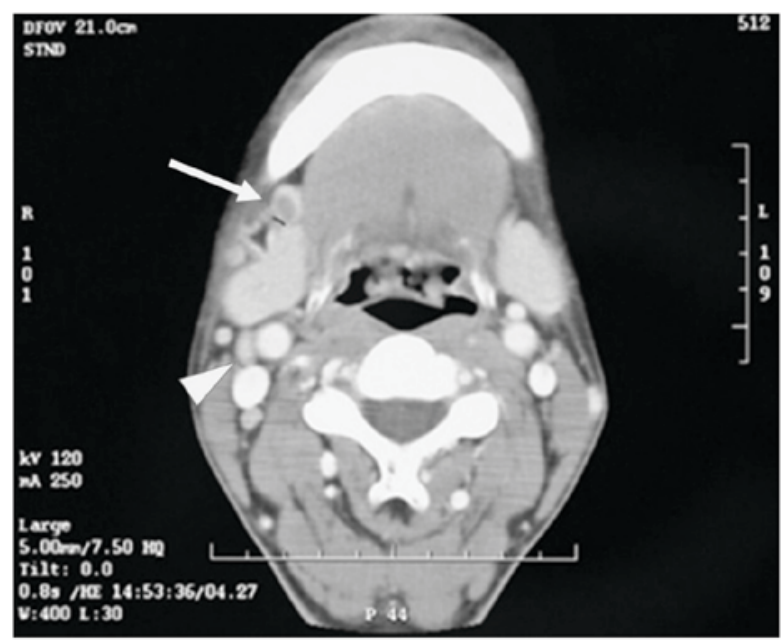

Figure 2. Computed tomography revealed a $1.2 \times 0.9 \mathrm{~cm}$ mass with rim enhancement and a central necrotic lesion in the level I lymph node (indicated by the arrow). This was identified histologically as lymph node metastasis. Lymph node lesions $<1.0 \mathrm{~cm}$ in diameter in levels II and III with homogeneous contrast enhancement (indicated by the arrowhead) were diagnosed histologically as Kikuchi-Fujimoto disease. All of above lymph nodes were in the right neck region.

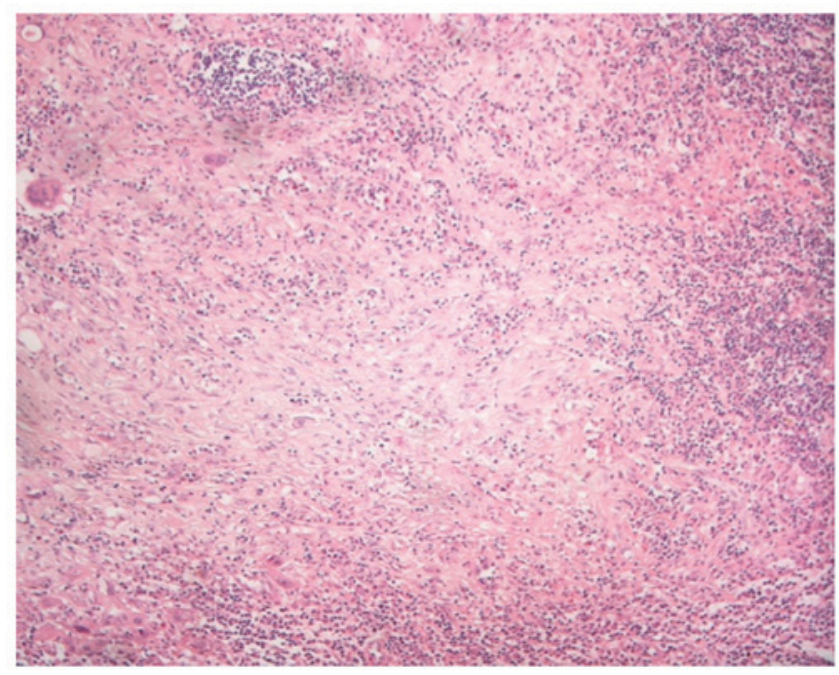

Figure 3. Histopathological examination revealed squamous cell carcinoma with one metastatic lymph node at level I, with necrotic lesions. Magnification, $\mathrm{x} 40$. 


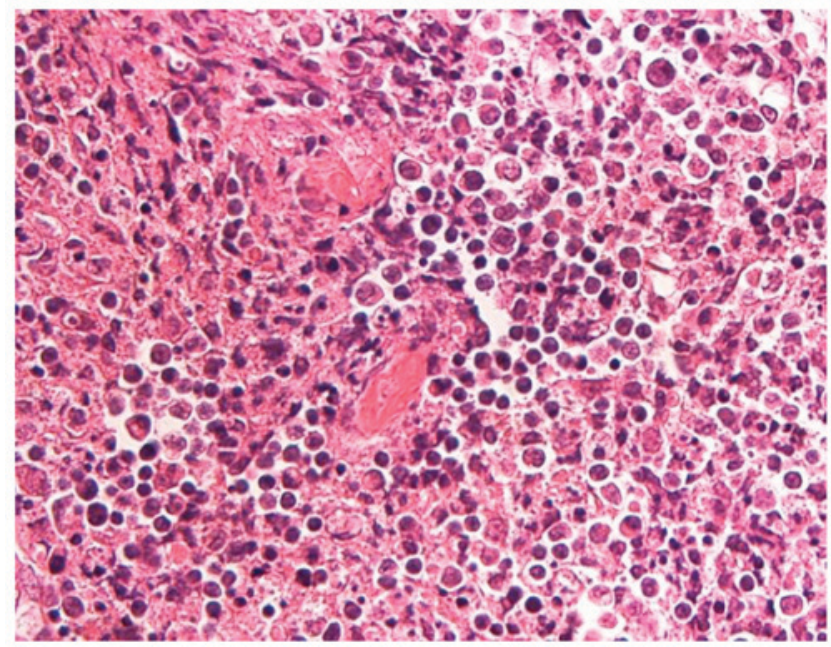

Figure 4. Histopathological examination revealed a Kikuchi-Fujimoto disease lymph node at level II with karyorrhectic foci and large numbers of histiocytes. Magnification, x400.

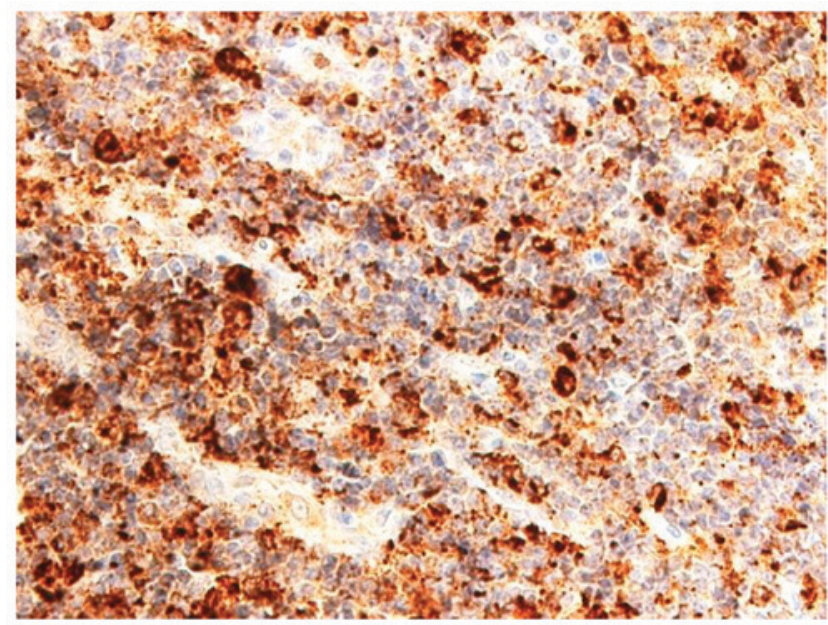

Figure 5. Histiocytes in the karyorrhectic foci of the Kikuchi-Fujimoto disease lesion were positive for cluster of differentiation 68. Magnification, $\mathrm{x} 200$.

histiocytes (Fig. 5). Based on histological and immunohistochemical findings it was therefore concluded that the level II and III LN lesions were KFD (lymphohistiocytic type).

The patient remained disease-free for 6 years after the initial surgery, following which the right side of the patient's posterior neck became swollen, and CT revealed multiple swollen posterior LNs, including spinal accessory nodes outside the scope of the previous neck dissection. High 2-[18F]-fluoro-2deoxy-D-glucose (FDG) uptake by these LNs was demonstrated by FDG positron emission tomography (FDG-PET)/CT (Fig. 6). No axillary or inguinal lymphadenopathy was found, and a second primary tumor of the neck or recurrent KFD was suspected. To diagnose the lesion clearly, an excision biopsy (July 2014) and subsequent histological examination of six posterior cervical LNs was performed, with the results suggesting KFD (Fig. 7; a 3- $\mu$ m section was cut and stained with hematoxylin and eosin and evaluated with brightfield microscopy). No malignant lesions, including regional recurrent SCC or lymphoma, were detected pathologically. No

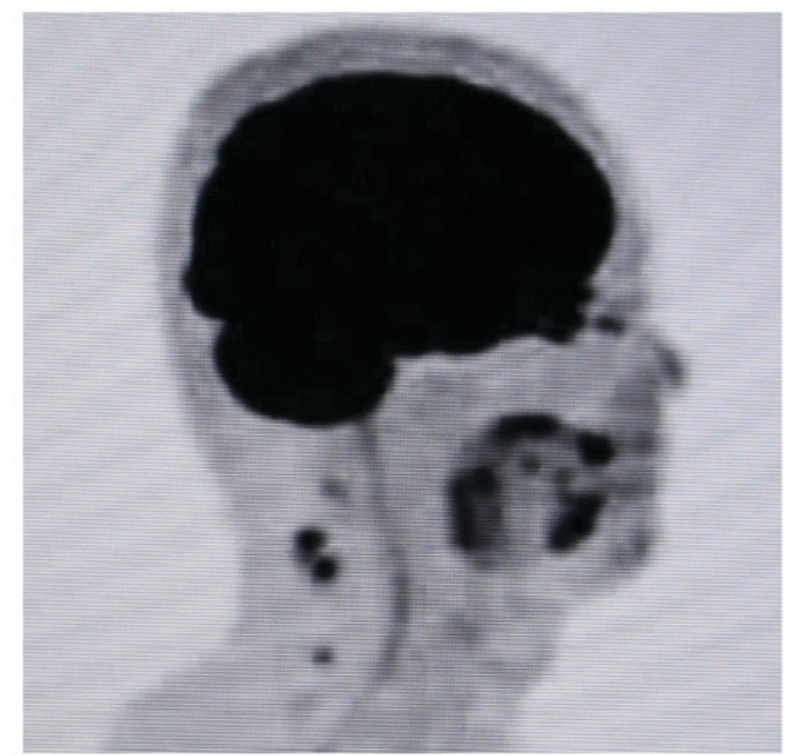

Figure 6. FDG-positron emission tomography revealed FDG uptake in the right posterior cervical region. FDG, 2-[18F]-fluoro-2-deoxy-D-glucose.

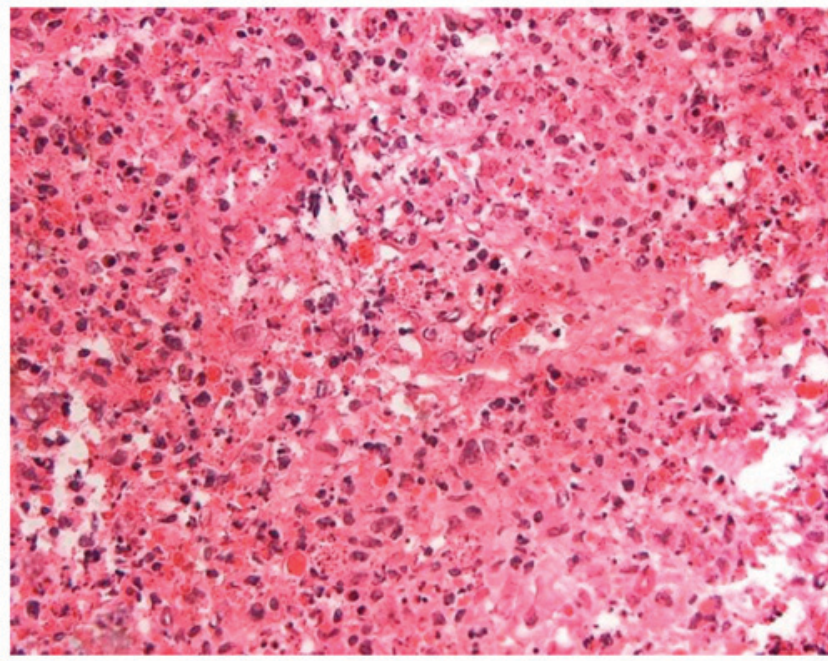

Figure 7. Histological findings following lymph node excision suggested Kikuchi-Fujimoto disease. Magnification, x200.

preoperative or postoperative therapy was performed before or after the second LN excision (July 2014). The residual lymphadenopathy gradually resolved on palpation and postoperative CT (5 months after surgery) confirmed the disappearance of the lymphadenopathy without treatment [Lee et al (12) described the resemble symptom]. Therefore, based on clinical and pathological findings, the lesion was diagnosed as recurrent KFD. The patient remained well, with no clinical or radiological signs of recurrence or metastasis, for 17 months after the second LN excision (July 2014).

\section{Discussion}

To the best of our knowledge, this is the first reported case of a patient with simultaneous tongue cancer, regional $\mathrm{LN}$ metastasis and KFD. This highlights the need for clinicians to consider KFD when they encounter LNs with necrotic lesions 
Table I. Reports of patients with KFD associated with cancer.

\begin{tabular}{|c|c|c|c|c|c|c|c|}
\hline $\begin{array}{l}\text { Case } \\
\text { no. }\end{array}$ & Age & Sex & Type of cancer & $\begin{array}{c}\text { Metastatic } \\
\text { LN } \\
\text { presence }\end{array}$ & LN site of KFD & $\begin{array}{c}\text { Recurrence } \\
\text { of } \\
\text { KFD }\end{array}$ & $\begin{array}{c}\text { (Refs.) } \\
\text { no. }\end{array}$ \\
\hline 1 & 57 & Female & $\begin{array}{l}\text { Recurrent malignant fibrous } \\
\text { histiocytoma of thigh }\end{array}$ & $(-)$ & Inguinal (regional) & $(-)$ & (18) \\
\hline 2 & 37 & Male & Stomach adenocarcinoma & $(-)$ & Cervical & $(-)$ & (19) \\
\hline 3 & 66 & Female & Invasive lobular carcinoma of breast & $(-)$ & Axillary (regional) & $(-)$ & (20) \\
\hline 4 & 27 & Female & $\begin{array}{l}\text { Remission period of diffuse } \\
\text { large B-cell lymphoma }\end{array}$ & $(-)$ & Cervical & $(-)$ & (21) \\
\hline 5 & 30 & Female & $\begin{array}{l}\text { Remission period of diffuse large } \\
\text { B-cell lymphoma }\end{array}$ & $(-)$ & Axillary & $(-)$ & (21) \\
\hline 6 & NA & Male & Buccal mucosa cancer & $(+)$ & Cervical (regional) & $(-)$ & (22) \\
\hline 7 & 41 & Female & $\begin{array}{l}\text { Post-treatment of thyroid cancer } \\
\text { and cervical metastasis }\end{array}$ & $(-)$ & Cervical (regional) & NA & (23) \\
\hline 8 & 58 & Male & Prostate cancer (postoperative) & $(-)$ & Cervical & $(-)$ & (24) \\
\hline 9 & NA & NA & NA & NA & NA & NA & (25) \\
\hline 10 & NA & NA & NA & NA & NA & NA & (25) \\
\hline 11 & NA & NA & Resolved lymphoma & $(-)$ & NA & $(-)$ & (26) \\
\hline 12 & 37 & Female & Melanoma of thigh & $(+)$ & Inguinal (regional) & $(-)$ & (27) \\
\hline 13 & 38 & Male & $\begin{array}{l}\text { Regional recurrence of } \\
\text { papillary thyroid carcinoma }\end{array}$ & $(+)$ & Cervical (regional) & $(-)$ & (28) \\
\hline 14 & 48 & Male & Tongue SCC & $(+)$ & Cervical (regional) & $(+)$ & $\begin{array}{l}\text { Current } \\
\text { study }\end{array}$ \\
\hline
\end{tabular}

LN, lymph node; KFD, Kikuchi-Fujimoto disease; NA, not available; SCC, squamous cell carcinoma.

and without cancerous cells, and indicates that a combination of clinical and pathological assessments may aid in the identification of KFD, in addition to ruling out metastasis in initial and recurrent lymphadenopathies. This is also the first report, to the best of our knowledge, of recurrent KFD on the same side as that of the original lesion (metastasis and KFD), and presents the oldest reported patient with recurrent KFD, occurring at 54 years old (initial presentation, 48 years old). Prior to our case, the oldest patient of recurrent KFD was 50 years old (13).

The literature from 1972-2016 was searched using PubMed and Google Scholar with the following key words or phrases: 'Kikuchi's disease AND cancer', 'Kikuchi's disease AND malignancy', 'Kikuchi-Fujimoto disease AND cancer', 'Kikuchi-Fujimoto disease AND malignancy', 'histiocytic necrotizing lymphadenitis AND cancer', 'or' 'histiocytic necrotizing lymphadenitis AND malignancy.' This led to the identification of 13 cases of patients with KFD and cancer (14-24) (Table I). However, none of the previously reported cases had recurrent KFD. Three reports in the English literature-described metastatic LNs together with KFD lesions $(18,23,24)$, where KFD was detected incidentally during cancer treatment. Cancer clinicians often assume that regional $\mathrm{LN}$ swelling is due to metastasis, and fail to consider the possibility of KFD.

The etiology of KFD remains unknown (25). Although various factors, including Yersinia enterocolitica, Toxoplasma gondii, Epstein-Barr virus, human herpes virus types 6, 7 and 8 , systemic lupus erythematosus, Hashimoto's thyroiditis, silicone breast implants and pacemaker implantation have been implicated in its development (26-30), it remains unclear if these are causative factors or coincidental phenomena. Cancer may also serve a role in the development of KFD, and is the most important factor for clinicians to be aware of. Kikuchi (1) and Fujimoto et al (2) pointed out the potential for the misdiagnosis of KFD as cancer metastasis in 1972, but few reports have described metastasis as a differential diagnosis of KFD $(18,21)$, and only three reports have described metastatic LNs together with KFD lesions $(18,23,24)$. To the best of our knowledge, the current report is the first to describe methods for differentiating between these two diseases. The results of the present report lead to the hypothesis that previous cases of KFD may have been misdiagnosed as metastasis in patients with cancer, thus leading to the underestimation of its incidence. National Comprehensive Cancer Network guidelines (31) suggest that risk factors, including multiple metastasis positive nodes, N2 or N3 nodal disease, and nodal disease in levels IV or V, should be considered as indications for postoperative therapy in patients with head and neck cancer, indicating that the misdiagnosis of KFD may lead to unnecessary adjuvant therapy.

The detection of LNs with necrotic lesions with no clinical or pathological indication of cancerous cells should alert clinicians to the possibility of KFD. The case reported in the present study exhibited apparent SCC in level I, but necrotic lesions without cancerous cells in levels II and III. Although the level II and III lesions were ultimately diagnosed as KFD 
by immunohistochemical examination, it was essential to rule out the possibility of metastases, given that necrotic lesions lacking cancerous cells, but within the regional LN area of the cancer, may also represent a pathologic complete response, defined as no cancer lesion remaining after therapy (11). Necrosis can be determined clinically, as well as pathologically, and KFD LNs with necrosis may be misdiagnosed as metastases in patients with head and neck cancer.

Clinically, initial preoperative $\mathrm{CT}$ in the case discussed in the present report revealed central necrosis in the level I metastatic LN and homogeneous contrast enhancement in the level II and III KFD LNs. However, Kwon et al (32) reported that KFD LNs frequently exhibit central necrosis and homogeneous contrast enhancement CT patterns, suggesting that the CT appearance of KFD can be variable and mimic metastasis (33); therefore, KFD cannot be diagnosed clinically on the basis of CT alone. All three LN lesions in the case discussed in the present report were subsequently found to be necrotic pathologically; however, $\sim 50 \%$ of metastatic nodes also have necrotic lesions (34). The capability of MRI and US to differentiate between KFD necrotic lesions and metastases are also controversial (33,35-37).

The results of previous studies suggest that it is difficult to distinguish KFD from metastasis clinically on the basis of physical findings, imaging or time to recurrence. With regard to physical findings, our patient's initial KFD LNs were $\geq 1.0 \mathrm{~cm}$ in diameter. However, a firm/hard consistency, the absence of tenderness and a diameter $\geq 1.0 \mathrm{~cm}$ on palpation are symptoms of suspected metastasis (11,38-40). While KFD lymphadenopathy may be firm and sometimes painful (41), several studies have described KFD lesions as firm or non-tender (41-43). Kwon et al (32) reported that the mean maximum diameter of the affected cervical LNs was $1.62 \mathrm{~cm}$ in 1,196 KFD LNs from 96 patients (32). Furthermore, KFD LNs may be $>2.5 \mathrm{~cm}(4,43)$. KFD LNs may thus resemble metastatic lesions, based on palpation, though clinical palpation of the neck has been reported to be unreliable (44). KFD also has extranodal manifestations that mimic malignant LN disease, including extracapsular nodal spread of cancer metastasis $(9,45)$. KFD can occur in all levels of the cervical LNs (32). Cervical LNs affected by KFD are commonly located in the posterior cervical triangle $(18,32)$, but the submandibular nodes (levels I and II; oral cavity, oropharynx, hypopharynx, larynx) may also be affected, in addition to being the regional LNs involved in head and neck cancer $(32,46)$.

No definitive imaging modality for distinguishing between KFD and malignancy has yet been identified (47). US findings in KFD tend to be suspicious of malignancy, with findings including a round shape, gross necrosis and a loss of central fatty hila $(36,37)$. Youk et al (48) also indicated that KFD tended to be misdiagnosed as malignancy on the basis of US findings. Furthermore, FDG uptake in FDG-PET/CT is not specific for cancer $(4,47)$. Regarding time to recurrence, KFD typically recurs within 1 year of its initial resolution $(5,49)$, while LN metastasis also occurs within 1 year (50). Time to recurrence is therefore not diagnostic, as KFD recurrence and LN metastasis may occur at any time $(51,52)$.

Unexpected findings are encountered during pathologic examination in $>3 \%$ of neck dissections (53); however, the presence of metastatic LNs remains the most important finding for clinicians. Effective communication between the clinician and pathologist is needed to coordinate the clinical and pathologic characteristics needed to make an accurate diagnosis of KFD (18). The combination of clinical and pathological features may facilitate the diagnosis of KFD and rule out metastasis in initial and recurrent lymphadenopathies. In the case discussed in the present report, pathological findings supported the sparse clinical findings to lead to the diagnosis of KFD. However, for the recurrent lesions, the pathological findings were inconclusive, while the clinical findings of resolution of the lymphadenopathy on palpation and CT confirmed a diagnosis of KFD. The initial KFD was diagnosed pathologically by immunohistochemical examination of AE1/AE3, CD3, CD20 and CD68. In the case of the recurrent lesions, although pathological diagnosis suggested possible recurrent KFD based on the presence of histiocytes with nuclear debris, as observed in the initial KFD lesion, the lesion was too small to make a definitive diagnosis. However, following surgery, lymphadenopathy of the residual posterior LNs that resolved upon palpation and postoperative CT ( 5 months after surgery) confirmed a diagnosis KFD. Although the cytological and histological examinations were not conclusive in a study by Lee et al (12), the occurrence of enlarged LNs as recurrent KFD was diagnosed based on clinical findings, including complete resolution of lymphadenopathy on CT performed 1 month after LNs enlarged. In addition, KFD is difficult to diagnose with fine-needle aspiration (13,54-57). Although recurrent lymphadenopathy suggests a diagnosis of KFD on clinical grounds, this may not be confirmed by pathological examination following LN excision $(13,51)$, and some studies have reported a diagnosis of recurrent KFD based on clinical findings alone, without rebiopsy $(3,13,42,51,58)$. The diagnostic criteria for recurrent KFD are therefore obscure. In the current case, pathological examination was performed to rule out a second primary tumor. However, when the pathological results are inconclusive for KFD, as in the current case, postoperative follow-up and a CT scan may be required to confirm a diagnosis. Notably, KFD can recur 18-19 years after its initial presentation, indicating the need for long-term follow-up $(51,52)$.

To the best of our knowledge, this is the first report of a patient with simultaneous tongue cancer, regional LN metastasis and KFD. The results of the present study and previous studies indicate that clinicians should consider KFD when they encounter LNs with necrotic lesions without cancerous cells. A combination of clinical and pathological techniques may aid in the diagnosis of KFD, in addition to ruling out metastasis or a second primary cancer in initial and recurrent lymphadenopathies. In particular, careful attention should be paid to a lack of cancerous cells in necrotic LNs in patients with head and neck cancer, given that a misdiagnosis of KFD as metastasis may lead to unnecessary adjuvant therapy.

\section{Acknowledgements}

The authors would like to thank Dr Takeaki Tomoyose (Division of Endocrinology, Diabetes and Metabolism, Hematology and Rheumatology, Second Department of Medicine, Graduate School of Medicine, University of the Ryukyus, Nishihara, 
Japan) who contributed to the patient diagnosis by excluding malignant lymphoma. The authors would also like to thank the staff of Edanz Group Japan for providing editing assistance for this manuscript.

\section{References}

1. Kikuchi M: Lymphadenitis showing focal reticulum cell hyperplasia with nuclear debris and phagocytes: A clinicopathological study. Acta Hematol Jpn 35: 379-380, 1972 (In Japanese).

2. Fujimoto Y, Kozima Y and Yamaguchi K: Cervical subacute necrotizing lymphadenitis: a new clinicopathologic entity. Naika 20: 920-927, 1972 (In Japanese).

3. Kim TY, Ha KS, Kim Y, Lee J, Lee K and Lee J: Characteristics of Kikuchi-Fujimoto disease in children compared with adults. Eur J Pediatr 173: 111-116, 2014

4. Ranabhat S, Tiwari M, Kshetri J, Maharjan S and Osti BP: An uncommon presentation of Kikuchi Fujimoto disease: A case report with literature review. BMC Res Notes 8: 478, 2015.

5. Dumas G, Prendki V, Haroche J, Amoura Z, Cacoub P, Galicier L, Meyer O, Rapp C, Deligny C, Godeau B, et al: Kikuchi-Fujimoto disease: Retrospective study of 91 cases and review of the literature. Medicine (Baltimore) 93: 372-382, 2014.

6. Kim JE, Lee EK, Lee JM, Bae SH, Choi KH, Lee YH, Hah JO, Choi JH, Kong EJ and Cho IH: Kikuchi-Fujimoto disease mimicking malignant lymphoma with $2-[(18) \mathrm{F}]$ fluoro-2-deoxy-D-glucose PET/CT in children. Korean J Pediatr 57: 226-231, 2014.

7. Dalton J, Shaw R and Democratis J: Kikuchi-Fujimoto disease. Lancet 383: 1098, 2014.

8. Sobin LH and Wittekind C: Head and neck tumours. In: TNM Classification of Malignant Tumours. 6th edition. John Wiley \& Sons, Hoboken, NJ, pp19-56, 2002.

9. Sturgis EM, Moore BA, Glisson BS, Kies MS, Shin DM and Byers RM: Neoadjuvant chemotherapy for squamous cell carcinoma of the oral tongue in young adults: A case series. Head Neck 27: 748-756, 2005.

10. Licitra L, Grandi C, Guzzo M, Mariani L, Lo Vullo S, Valvo F, Quattrone $\mathrm{P}$, Valagussa $\mathrm{P}$, Bonadonna G, Molinari R and Cantù G: Primary chemotherapy in resectable oral cavity squamous cell cancer: A randomized controlled trial. J Clin Oncol 21: 327-333, 2003.

11. Eisenhauer EA, Therasse P, Bogaerts J, Schwartz LH, Sargent D, Ford R, Dancey J, Arbuck S, Gwyther S, Mooney M, et al: New response evaluation criteria in solid tumours: Revised RECIST guideline (version 1.1). Eur J Cancer 45: 228-247, 2009.

12. Lee SK, Bahn YE and Kim DE: Features of sequential CT and US-guided biopsy in recurrent Kikuchi disease of the neck: a Case report. Ear Nose Throat 92: 442-448, 2013.

13. Bogusz AM and Bhargava P: Recurrent histiocytic necrotizing lymphadenitis with a long latency in a patient with autoimmunity: A case report and review of literature. Int J Surg Pathol 21: 287-296, 2013.

14. Chan JK and Ng CS: Kikuchi's histiocytic necrotizing lymphadenitis in the regional lymph nodes of malignant fibrous histiocytoma: Causal or coincidental? Histopathology 12: 448-451, 1988.

15. Radhi JM, Skinnider L and McFadden A: Kikuchi's lymphadenitis and carcinoma of the stomach. J Clin Pathol 50: 530-531, 1997.

16. Aqel NM and Peters EE: Kikuchi's disease in axillary lymph nodes draining breast carcinoma. Histopathology 36: 280-281, 2000.

17. Yoshino T, Mannami T, Ichimura K, Takenaka K, Nose S, Yamadori I and Akagi T: Two cases of histiocytic necrotizing lymphadenitis (Kikuchi-Fujimoto's disease) following diffuse large B-cell lymphoma. Hum Pathol 31: 1328-1331, 2000.

18. Lin HC, Su CY, Huang CC, Hwang CF and Chien CY: Kikuchi's disease: A review and analysis of 61 cases. Otolaryngol Head Neck Surg 128: 650-653, 2003.

19. Dequanter D, Lothaire, Haller, Saint Aubain Somerhausen and Andry: Kikuchi-Fujimoto disease mimicking thyroid metastasis. Rev Stomatol Chir Maxillofac 106: 302-303, 2005 (In French).

20. Waters A, Williams C and Pal A: Kikuchi Disease: A unique case of fever of unknown origin. Kansas J Med 6: 60-64, 2013.
21. Yu SC, Chen CN, Huang HI, Chen TC, Wang CP, Lou PJ, Ko JY, Hsiao TY and Yang TL: Diagnosis of Kikuchi-Fujimoto disease: A comparison between open biopsy and minimally invasive ultrasound-guided core biopsy. PLoS One 9: e95886, 2014.

22. Yoo IH, Na H, Bae EY, Han SB, Lee SY, Jeong DC and Kang JH: Recurrent lymphadenopathy in children with Kikuchi-Fujimoto disease. Eur J Pediatr 173: 1193-1199, 2014.

23. Urbanellis P, Chin-Lenn L, Teman CJ and McKinnon JG: Kikuchi-Fujimoto lymphadenitis imitating metastatic melanoma on positron emission tomography: A case report. BMC Surg 15: 50, 2015.

24. Park JJ, Seo YB, Choi HC, Kim JW, Shin MK, Lee DJ and Lee J: Kikuchi-Fujimoto disease coexistent with papillary thyroid carcinoma in a single lymph node. Soonchunhyang Med Sci 21: 10-14, 2015.

25. Sharma V and Rankin R: Fatal Kikuchi-like lymphadenitis associated with connective tissue disease: A report of two cases and review of the literature. Springerplus 4: 167, 2015.

26. Deaver D, Horna P, Cualing H and Sokol L: Pathogenesis, diagnosis, and management of Kikuchi-Fujimoto disease. Cancer Control 21: 313-321, 2014.

27. Lee DH, Lee JH, Shim EJ, Cho DJ, Min KS, Yoo KY and Min K: Disseminated Kikuchi-Fujimoto disease mimicking malignant lymphoma on positron emission tomography in a child. J Pediatr Hematol Oncol 31: 687-689, 2009.

28. Rubio SI, Plewinsky TS, Sabatini M and Poretsky L: Kikuchi's disease associated with Hashimoto's thyroiditis. J Endocrinol Invest 19: 136-137, 1996.

29. Sever CE, Leith CP, Appenzeller J and Foucar K: Kikuchi's histiocytic necrotizing lymphadenitis associated with ruptured silicone breast implant. Arch Pathol Lab Med 120: 380-385, 1996.

30. Charalabopoulos K, Charalabopoulos A, Binolis J, Papalimneou V and Ioachim E: Is implant pacemaker a physicochemical cause triggering Kikuchi-Fujimoto disease? In Vivo 16: 73-76, 2002.

31. National Comprehensive Cancer Network: Head and Neck Cancers Version 1. https://www.nccn.org/professionals/physician_gls/pdf/head-and-neck.pdf. Accessed June 8, 2015.

32. Kwon SY, Kim TK, Kim YS, Lee KY, Lee NJ and Seol HY: CT findings in Kikuchi disease: Analysis of 96 cases. AJNR Am J Neuroradiol 25: 1099-1102, 2004.

33. Na DG, Chung TS, Byun HS, Kim HD, Ko YH and Yoon JH: Kikuchi disease: CT and MR findings. AJNR Am J Neuroradiol 18: 1729-1732, 1997.

34. King AD, Tse GM, Ahuja AT, Yuen EH, Vlantis AC, To EW and van Hasselt AC: Necrosis in metastatic neck nodes: Diagnostic accuracy of CT, MR imaging, and US. Radiology 230: 720-726, 2004.

35. Sumi M and Nakamura T: Diagnostic importance of focal defects in the apparent diffusion coefficient-based differentiation between lymphoma and squamous cell carcinoma nodes in the neck. Eur Radiol 19: 975-981, 2009.

36. Yoo JL, Suh SI, Lee YH, Seo HS, Kim KM, Shin BK, Song JY and Seol HY: Gray scale and power Doppler study of biopsy-proven Kikuchi disease. J Ultrasound Med 30: 957-963, 2011.

37. Lee KH and Ryu J: Real-time elastography of cervical lymph nodes in Kikuchi disease. J Ultrasound Med 33: 2201-2205, 2014.

38. Hay ID, Lee RA, Davidge-Pitts C, Reading CC and Charboneau JW: Long-term outcome of ultrasound-guided percutaneous ethanol ablation of selected 'recurrent' neck nodal metastases in 25 patients with TNM stages III or IVA papillary thyroid carcinoma previously treated by surgery and 131I therapy. Surgery 154: 1448-1455, 2013.

39. Lang S and Kansy B: Cervical lymph node diseases in children. GMS Curr Top Otorhinolaryngol Head Neck Surg 13: Doc08, 2014.

40. Cheng CY, Sheng WH, Lo YC, Chung CS, Chen YC and Chang SC: Clinical presentations, laboratory results and outcomes of patients with Kikuchi's disease: Emphasis on the association between recurrent Kikuchi's disease and autoimmune diseases. J Microbiol Immunol Infect 43: 366-371, 2010.

41. Tariq H, Gaduputi V, Rafiq A and Shenoy R: The enigmatic kikuchi-fujimoto disease: A case report and review. Case Rep Hematol 2014: 648136, 2014.

42. Phupong V and Poomtavorn Y: Kikuchi disease during pregnancy. Arch Gynecol Obstet 274: 393-396, 2006.

43. Nieman RB: Diagnosis of Kikuchi's disease. Lancet 335: 295, 1990. 
44. Langhans L, Bilde A, Charabi B, Therkildsen $\mathrm{MH}$ and von Buchwald C: Evaluation of sentinel lymph node size and shape as a predictor of occult metastasis in patients with squamous cell carcinoma of the oral cavity. Eur Arch Otorhinolaryngol 270: 249-254, 2013.

45. Thakral B, Zhou J and Medeiros LJ: Extranodal hematopoietic neoplasms and mimics in the head and neck: An update. Hum Pathol 46: 1079-1100, 2015.

46. Nakamura T and Sumi M: Nodal imaging in the neck: Recent advances in US, CT and MR imaging of metastatic nodes. Eur Radiol 17: 1235-1241, 2007.

47. Ito K, Morooka M and Kubota K: Kikuchi disease: 18F-FDG positron emission tomography/computed tomography of lymph node uptake. Jpn J Radiol 28: 15-19, 2010.

48. Youk JH, Kim EK, Ko KH and Kim MJ: Sonographic features of axillary lymphadenopathy caused by Kikuchi disease. J Ultrasound Med 27: 847-853, 2008.

49. Song JY, Lee J, Park DW, Sohn JW, Suh SI, Kim IS, Kim WJ, Kim MJ and Cheong HJ: Clinical outcome and predictive factors of recurrence among patients with Kikuchi's disease. Int J Infect Dis 13: 322-326, 2009.

50. Kim JW, Roh JL, Kim JS, Lee JH, Cho KJ, Choi SH, Nam SY and Kim SY: (18)F-FDG PET/CT surveillance at 3-6 and 12 months for detection of recurrence and second primary cancer in patients with head and neck squamous cell carcinoma. Br J Cancer 109: 2973-2979, 2013.
51. Smith KG, Becker GJ and Busmanis I: Recurrent Kikuchi's disease. Lancet 340: 124, 1992.

52. Kosch M, Hausberg M, Barenbrock M, Rahn KH and Kisters K Histiocytic, necrotizing lymphadenitis as rare cause of cervical lymphadenopathy and fever of unknown origin-a case of biopsy proven recurrence over 19 years. Eur J Haematol 63: 282-283, 1999.

53. Sheahan P, Hafidh M, Toner M and Timon C: Unexpected findings in neck dissection for squamous cell carcinoma: Incidence and implications. Head Neck 27: 28-35, 2005.

54. Park HS, Sung MJ, Park SE and Lim YT: Kikuchi-Fujimoto disease of 16 children in a single center of Korea. Pediatr Allergy Immunol 18: 174-178, 2007.

55. Phelan E, Lang E, Gormley P and Lang J: Kikuchi-Fujimoto disease: A report of 3 cases. Ear Nose Throat J 86: 412-413, 2007.

56. Tong TR, Chan OW and Lee KC: Diagnosing Kikuchi disease on fine needle aspiration biopsy: A retrospective study of 44 cases diagnosed by cytology and 8 by histopathology. Acta Cytol 45: 953-957, 2001.

57. Zar R and McClintock C: Kikuchi-Fujimoto disease: A case report and review of literature. Conn Med 70: 491-494, 2006.

58. Alijotas-Reig J, Casellas-Caro M, Ferrer-Oliveras R, Cabero-Roura L and Vilardell-Tarres $M$ : Recurrent Kikuchi-Fujimoto disease during pregnancy: Report of case evolving into systemic lupus erythematosus and review of published work. J Obstet Gynaecol Res 34: 595-598, 2008. 